\title{
THE PREDICTIVE VALUE OF PAIN EVENT-RELATED POTENTIALS FOR THE CLINICAL EXPERIENCE OF PAIN
}

\author{
HELEN VOSSEN \\ Department of Psychiatry and Psychology, Maastricht University, \\ P.O. Box 5800, 6202 Az Maastricht, The Netherlands \\ helen.vossen@gmail.com \\ JIM VAN OS \\ Department of Psychiatry and Psychology, Maastricht University, \\ P.O. Box 5800, 6202 Az Maastricht, The Netherlands \\ j.vanos@sp.unimaas.nl \\ HERMIE HERMENS \\ Roessigh Research and Development \\ Roessinghsbleekweg 33b, 7522 AH Enschede, The Netherlands \\ h.hermens@rrd.nl \\ RICHEL LOUSBERG \\ Department of Psychiatry and Psychology, Maastricht University, \\ P.O. Box 5800, 6202 Az Maastricht, The Netherlands \\ r.lousberg@pn.unimaas.nl
}

\begin{abstract}
Event-Related Potentials have been found to be related to subjective experience of experimental pain. But how are they related to the subjective experience of clinical pain? The current study investigated the predictive value of the pain ERP for the subjective experience of clinical pain. Event-Related Potentials in response to experimental pain were measured in 75 chronic low back pain sufferers. In addition a two-week registration was done of the amount of pain they experienced in daily life. The results demonstrate that the N2-component at $\mathrm{Cz}$ and $\mathrm{C} 4$ of painERP (contralateral to the side of the stimulation) were significant predictors of clinical pain, even stronger predictors that the accompanying subjective ratings of experimental pain. Thus, it seems promising to use Event-Related Potentials as a more objective measure to make predictions about a person's likely pain experience in daily life
\end{abstract}

Keywords: Event-Related Potentials; Chronic pain; predictive value 


\section{Introduction}

Many experimental studies have been conducted in order to examine individual differences in the experience of pain. An important advantage of experimental laboratory studies is that the environment can be controlled and thus more specific conclusions can be drawn. Intensity, duration and location of a pain stimulus typically are under experimental control. However, an important issue in experimental laboratory research is the difficulty in generalizing the results to clinical practice. Furthermore, quantitative psychophysiological measures such as the peak amplitudes of Event-Related Potentials are even more difficult to translate to subjective pain experience. Although research has demonstrated that ERP's can serve as a more objective measurement of pain compared to subjective pain ratings $[3,4,19]$ and evidence has been provided that specific peak components of the ERP significantly correlate with subjective ratings of pain [11], the question remains what exactly ERP measures tell us about the amount of pain that people experience in daily life. Can the pain ERP be used as a predictor for clinical pain?

To our knowledge, no studies have been performed that attempted to answer these questions. However, there have been some studies in that demonstrated the predictive value of ERPs for other clinical outcomes. Kemp and colleagues [12] found that, in combination with personality and cognitive factors, the left-lateralized P150 could predict the severity of non-clinical depression. Also, specific components of the ERP have been found to predict awakening in coma patients [6]. Furthermore, selected ERP time segments across different electrodes have proven to contain signal features that discriminate Alzheimer patients from control subjects with high sensitivity and specificity [5].

The current study was designed to measure pain responses in both a laboratory setting and in daily life, in a sample of patients with chronic low back pain. The aim of the study was twofold. First, the relation between subjective ratings of experimental pain and subjective ratings of clinical pain was examined. Second, it was investigated to what degree cortical processing of experimental pain can predict clinical pain in daily life. The hypotheses were that i) a positive association exists between subjective ratings of experimental pain and clinical pain and ii) persons showing stronger pain processing in the experimental setting show higher pain ratings in daily life. 


\section{Methods}

Approval has been obtained from the medical ethics committee of the Academic Hospital Maastricht, on January, 6th, 2005. All subjects gave their verbal and written informed consent prior to the experiment.

\subsection{Subjects}

Seventy-seven subjects with chronic non-specific low back pain were included. Chronic low back pain was specifically chosen since this is a highly prevalent chronic pain problem which is a clear recognizable complaint for patients. The latter was especially important because the patients were drawn from the general population (via advertisements distributed door to door). They were required to have low back pain for at least six months with no other interfering pain complaints. The low back pain complaints concerned non-malignant chronic pain. Furthermore, they did not use any analgesics or psychoactive drugs.

\subsection{Stimuli}

The stimuli used in the experimental part of the study were electrical pulses of ten milliseconds duration. These were administered intracutaneuously on the left middle finger. For each participant, five different intensities based on that participant's sensation and pain thresholds were administered. Of the five intensities, one was the pain threshold and the other four were defined relative to this pain threshold, namely $-50 \%,-25 \%$, $+25 \%$ and $+50 \%$ of the threshold range which was defined as the range between the sensation threshold and the pain threshold. The sensation threshold was determined by first administering stimuli at zero intensity and then gradually increasing the intensity until the stimuli were experienced consciously. Once experienced consciously (sensation threshold), stimuli were once again administered with an intensity that gradually increased from the sensation threshold until the stimuli were defined as painful by the participant (pain threshold). This procedure was repeated three times in order to generate a more reliable measurement.

\subsection{Rating paradigm}

The stimuli, described above, were presented using a rating paradigm [2]. The paradigm consisted of 150 stimuli. The five intensities mentioned above were presented semi-randomly. The inter-stimulus interval (ISI) 
ranged from 9 to 11 seconds. Subjects were asked to rate the intensity of each stimulus on a Numeric Rating Scale (NRS) from 0 to 100 , whereby 0 is no sensation and 100 is the most excruciating pain imaginable. The first stimulus of the paradigm was set at the pain threshold. As a reference, subjects were instructed that this intensity was ' 60 '.

\subsection{EEG recording}

All EEG recordings were conducted in an electrically- and sound-shielded cubicle $\left(3^{*} 4 \mathrm{~m}^{2}\right) . \mathrm{Ag} / \mathrm{AgCl}$ electrodes were placed on Fz, Cz, Pz, C3, C4, T3 and T4 using the international 10-20 system [10]. Impedances were kept below $5 \mathrm{k} \Omega$. A reference electrode was placed on each ear lobe. In order to control for possible vertical eye movements, an electro-oculogram (EOG) electrode was placed 1 centimeter under the midline of the right eye. A ground electrode was placed at Fpz. All electrodes were fixed using 10-20 conductive paste. Neuroscan 4.3 software was used for EEG recording.

\subsection{Personality}

Subjects were asked to complete the NEO Personality Inventory. The NEO-PI-R is based on a five-factor model of personality. The five personality factors measured are Neuroticism, Extraversion, Openness, Agreeableness and Conscientiousness. Validity and reliability measures of the NEO-PI-R are adequate [8].

\subsection{Booklets}

In order to measure pain in daily life, subjects completed a daily questionnaire containing 4 questions concerning their pain complaints, during a two week period. Subjects were instructed to answer the questions before bedtime. The 4 questions were:

(1) How much pain do you have at this moment? (pain),

(2) How is your mood at this moment? ( mood),

(3) How much physical and mental effort did your activities cost today? (effort),

(4) How much stress did you experience today? (stress). 
These questions where assessed with Visual Analogue Scales measuring $100 \mathrm{~mm}$. These scales have adequate reliability and validity $[9,13]$

\subsection{Procedure}

Before starting the experiment, subjects were informed about the purpose of the study. Subjects were told that they would undergo EEG-registration while they received electric shocks. After signing the informed consent form, subjects were asked to fill in the NEO-PI-R. Then, EEG electrodes were placed and the shock electrode was attached to the top of the left middle finger as described by Bromm and Meier [2]: a small opening in the upper layer of the skin was prepared using a dental gimlet. Care was taken that this procedure was not painful. In the prepared opening, a platinum electrode was placed and fixed with tape. Next, the sensation and pain threshold were determined and after that the Rating paradigm was initiated. After the experiment subjects returned home and started filling in the first booklet the day after the experiment.

\subsection{ERPS}

EEG was recorded with $1000 \mathrm{~Hz}$ sampling rate, using Neuroscan 4.3 software. Trials were selected from the continuous EEG, from $200 \mathrm{~ms}$ prior to the stimulus until $1500 \mathrm{~ms}$ post-stimulus. Data was offline filtered (bandpass $0-50 \mathrm{~Hz}$ ) and baseline-corrected. Trials with EOG activity exceeding $+75 \mathrm{~mA}$ and $-75 \mathrm{~mA}$ were excluded from the analyses.

\subsection{Statistical analyses}

Linear regressions were performed in which the mean pain score of the booklets rated over 14 days was used as dependent variable. The analyses were performed in three steps. First, significant covariates were determined. We hypothesized that the following covariates were of potential influence on the mean pain score: age, gender, mood, stress, effort (all measured from the booklets), duration of pain complaints and personality (five main factors: Neuroticism, Extraversion, Openness, Conscientiousness and Altruism). Second, the mean rating in response to experimental pain stimuli (NRS) was added as predictor of clinical pain. Third, in addition to the significant covariates, mean ERP peak amplitudes of the following components: 
N1 (20-55msec), P1 (56-95msec), N2 (96-145msec), P2 (146-300msec) were tested as predictors of clinical pain. These components have been shown to be associated with the processing of stimulus intensity $[3,4,11,21]$. Only the mean ERP measures of the strongest intensity were used in the analyses.

All statistical analyses were performed using SPSS 16.0 software. P-values $\leq 0.05$ were considered statistically significant. For the analyses concerning the ERP measures, the p-value was corrected for multiple testing $(0.05 / 3=0.017)$.

\section{Results}

Seventy-seven subjects ( 35 men and 42 women) with chronic low back-pain were included in the analyses. The mean pain score collected over 14 days was computed based on valid ratings only (sum/(14-number of missing values)). More than $83 \%$ of the subjects did not have any missing data. The mean pain score was normally distributed with an overall mean of $38.9(\mathrm{SD}=16.3)$. The mean age was 40.3 years $(\mathrm{SD}=15.2)$. Mean duration of the low back-pain complaints was 12.2 years $(\mathrm{SD}=11.9)$. Figure 1 shows the grand average of the response to the strongest pain intensity (50\% above the pain threshold) at Cz.

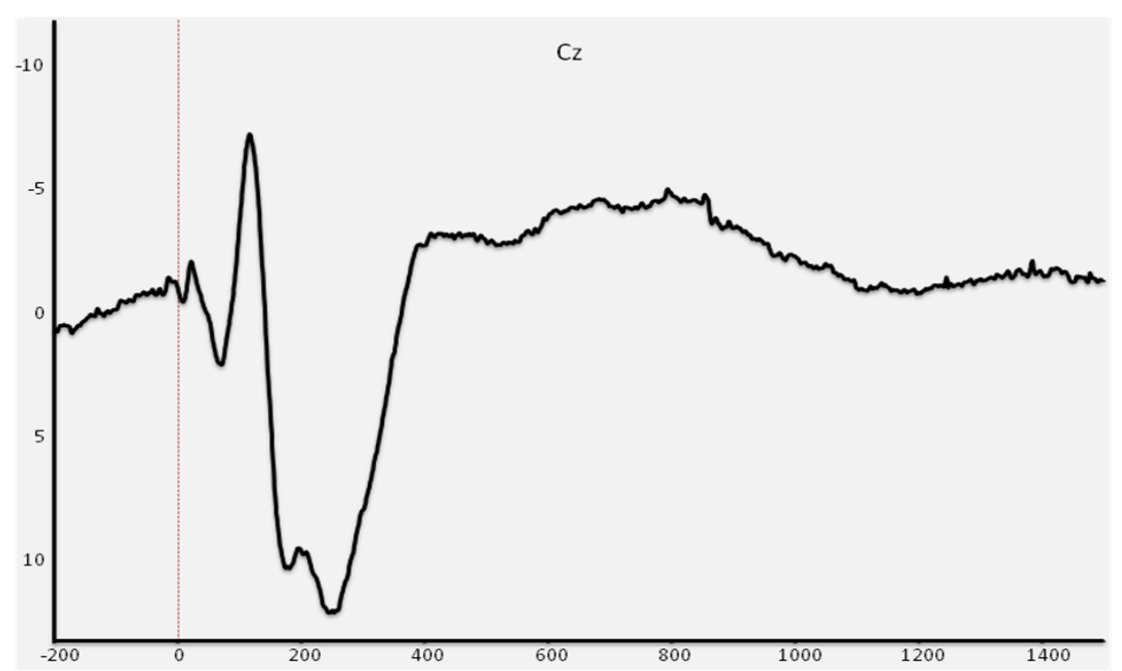

Fig. 1. Grand Average of the strongest pain intensity (50\% above the pain threshold) at $\mathrm{Cz}$. The $\mathrm{Y}$ axis represent amplitude in $\mu \mathrm{V}$ and the the $\mathrm{x}$-axis represents time in milliseconds.

First, covariates which significantly predicted the mean pain score were identified. A regression analysis with the mean clinical pain score as dependent variable and the list of covariates as independent variables (see 
method section), showed that only stress (as reported in the booklets) and conscientiousness (one of the NEOPI-R scales) were significant predictors. Stress was positively associated with the clinical pain score $(B=0.58$, $\mathrm{SE}=0.13, \mathrm{p}<0.001$ ), indicating that a higher stress score was associated with higher clinical pain scores. Conscientiousness was also positively associated with clinical pain $(\mathrm{B}=0.29, \mathrm{SE}=0.10, \mathrm{p}=0.003)$. Subjects who were more conscientious reported higher levels of clinical pain. This model explained $30 \%$ of the total variance of the mean pain score. The other covariates did not significantly predict clinical pain score (all $p$ values $>0.15)$.

In order to test the first hypothesis, a regression with stress, conscientiousness and the mean NRS as predictors was tested. This model demonstrated that subjective ratings of experimental pain stimuli significantly predicted subsequent ratings of clinical pain $(B=-0.23, \mathrm{SE}=0.11, \mathrm{p}=0.042)$. The Beta was negative, indicating that individuals with lower ratings of experimental pain reported stronger pain in dailylife. This model explained 34\% of the total variance of the mean pain score. Thus, hypothesis 1 was rejected. For the second hypothesis, the ERP measures were added instead of the subjective ratings of experimental pain. Separate models were tested for each ERP component (4), and locations were clustered: the central midline locations $(\mathrm{Fz}, \mathrm{Cz}, \mathrm{Pz})$, the lateral locations $(\mathrm{C} 3, \mathrm{C} 4, \mathrm{~T} 3, \mathrm{~T} 4)$ and the sensory motor cortex locations $(\mathrm{C} 3, \mathrm{Cz}, \mathrm{C} 4)$. The results of the series of regression analyses yielded one specific significant model with significant ERP predictors. This model consisted of the psychological predictors in combination with the N2component at $\mathrm{Cz}$ and $\mathrm{C} 4$ (table 1). This model explained 37\% of the total variance of the mean pain score, an increase of $7 \%$ compared to the model with only the covariates. The two N2-components had directionally opposite $\mathrm{B}$ estimates, the $\mathrm{B}$ of the $\mathrm{N} 2$-component at $\mathrm{Cz}$ being negative and the $\mathrm{B}$ of the $\mathrm{N} 2$-component at $\mathrm{C} 4$ being positive. Further analysis revealed that the two N2-components were only statistically significant when they were both in the model. This model explained 39\% of the total variance of the mean pain score.

Finally, the model with significant ERP measures was extended with the mean NRS score (table 2). This model revealed that the mean NRS was no longer a significant predictor of clinical pain when corrected for the cortical processing of experimental stimuli. The N2-components of $\mathrm{Cz}$ and $\mathrm{C} 4$, however, did remain statistically significant, indicating their effect was not reducible to other predictors. 
Table 1 Model with only ERP predictors.

\begin{tabular}{llrrr}
\hline Model & \multicolumn{2}{c}{ Unstandardized Coefficients } & \multirow{2}{*}{ Sig. } \\
& & B & \multicolumn{2}{c}{ Std. Error } \\
\hline \\
stress & .668 & .126 & .000 \\
conscientiousness & .256 & .095 & .009 \\
CzN2 & -1.472 & .508 & .005 \\
C4N2 & 1.750 & .675 & .012 \\
\hline
\end{tabular}

Table 2 Model with ERP predictors and mean NRS.

\begin{tabular}{llccc}
\hline Model & & \multicolumn{2}{c}{ Unstandardized Coefficients } & \multicolumn{1}{l}{ Sig. } \\
& & B & \multicolumn{2}{c}{ Std. Error } \\
\hline & stress & .671 & .125 & .000 \\
conscientiousness & .270 & .094 & .005 \\
CzN2 & -1.348 & .508 & .010 \\
C4N2 & 1.599 & .673 & .020 \\
mean NRS & -.179 & .111 & .110 \\
\hline
\end{tabular}

\section{Discussion}

The aim of the current study was to investigate whether Event-Related Potentials in response to experimental noxious stimuli predicted the level of clinical pain in daily life. The results demonstrated that this was the case.

Since multiple factors influence the subjective experience of clinical pain, significant covariates were determined before the hypotheses were tested. Stress, which was also measured in daily life, was the covariate with the strongest association. Higher stress scores were associated with higher clinical pain ratings. This finding is in accordance with findings of previous studies [1,14]. Furthermore, the personality trait conscientiousness was also positively associated with ratings of clinical pain. Conscientiousness is a personality trait which is characterized by self discipline, carefulness, deliberation and need for achievement [8]. Subjects who were more conscientious reported more clinical pain. This is in agreement, to a degree, with an earlier study by Farman and colleagues [7], who demonstrated that chronic pain patients showed higher scores on the conscientiousness scale compared to pain free controls. All other hypothesized covariates were not associated with clinical pain ratings. 
Subsequently, the association between subjective ratings of experimental pain (NRS) and ratings of clinical pain was tested. The results showed that, in combination with the two covariates, higher ratings of clinical pain were associated with lower ratings of experimental pain. This was not as hypothesized: subjective response to experimental pain and clinical pain co-vary in opposite rather than similar directions. A possible post-hoc explanation may be that persons experiencing higher levels of pain in daily life are "desensitized" to experimental nociceptive stimuli.

Next, the association between cortical processing of experimental pain and ratings of clinical pain was analyzed. Independent of the covariates stress and conscientiousness, the $\mathrm{N} 2$-component at $\mathrm{Cz}$ and $\mathrm{C} 4$ were significant predictors of the mean rating of clinical pain. The N2-component at $\mathrm{Cz}$ was negatively associated with clinical pain whereas the $\mathrm{N} 2$-component at $\mathrm{C} 4$ (which is contralateral to the side of stimulation) was positively associated with clinical pain. The fact that these components were only significant in the presence of each other suggests 'suppression'. Each of these two ERP measures may explain a small but unique part of the mean pain variance, and co-depend on each other in the model. The fact that these two measures have directionally opposite associations, is interesting since it was expected that a stronger N2-component was associated with more pain in daily-life (the more negative the N2-component the higher the mean pain score). This only holds true for the N2-component at Cz. The opposite was observed for the N2-component at C4, where more negative ERP amplitudes correspond with lower mean pain ratings. This suggests an association corresponding to pain reduction for the N2-component at C4 and one suggesting increase in pain for the N2componenent at $\mathrm{Cz}$. The phenomenon of inhibitory and excitatory factors influencing the pain experience has been described earlier [20].

Finally, analyses in which mean NRS as well as the ERP measures were included revealed that the ERP measures remained significant predictors whereas the subjective NRS did not. This finding suggests that ERP measures may be useful for prediction of chronic pain over-and-above subjective ratings, possibly because Event-Related Potentials are less vulnerable to bias and are multidimensional measures (measuring more aspects of the processing of pain).

This study has some limitations. First, pain in daily life was measured only once a day, at a fixed time. Perhaps multiple random measurements during the day, for example using momentary assessment technology [16], provides more detailed information about pain and its course during the day. Second, a possible 
important covariate of daily pain ratings, which was not measured in this study, is the presence of a partner or spouse during the research interview. Research has revealed a significant pain augmenting influence of solicitous responses of spouses $[15,18]$. Including this covariate in the analyses could perhaps explain more error variance of mean pain score and have a positive effect on the explanatory capacity of the ERP measures. Finally, we performed 12 regression analyses in order to test the hypotheses related to ERP measures. Pvalues were only corrected for the clusters of cranial locations. Perhaps this does not lower the type I error enough. However, a strict Bonferroni correction increases the type II error substantially, which could result in "throwing the child out with the bathwater" [17].

This study demonstrates that the pain ERP measured in an experimental laboratory setting has predictive value for clinical pain in daily life. Although the contribution of the ERP measures to the explanation of the mean pain variance may be considered relatively small (7\%), the fact that a significant contribution to pain in daily life up to two weeks later could be demonstrated may be considered remarkable. Thus, the two ERP components in questions may be investigated further for use as an objective measure to make predictions about a person's likely pain experience in daily life.

\section{References}

[1] Aslaksen PM, Flaten MA, The roles of physiological and subjective stress in the effectiveness of a placebo on experimentally induced pain. Psychosom Med 70: 811-818, 2008.

[2] Bromm, B, Meier W, The intracutaneous stimulus: a new pain model for algesimetric studies. Meth Find in Exp Clin Pharmacol 6: 405-410,1984.

[3] Bromm B (1984) Pain-related components in the cerebral potential: experimental and multivariate statistical approaches. In Pain measurement in man: Neurophysiological correlates of pain (Ed. Bromm B), pp. 257-290. Elsevier: Amsterdam.

[4] Becker DE, Haley DW, Urena VM, Yingling CD, Pain measurement with evoked potentials: combination of subjective ratings, randomized intensities, and long interstimulus Interval procedures a P300-like confound. Pain 84: $37-47,2000$.

[5] Benvenuto J, Jin Y, Casale M, Lynch G, Granger R, Identifaction of diagnostic evoked response potentials segments in Alzheimer's disease. Exp Neurol 176: 269-276, 2002. 
[6] Daltrozzo J, Wioland N, Mutschler V, Kotchoubey B, Predicting coma and other low responsive patient's outcome using event-related brain potentials: a meta-analyses. Clin Neurophysiol 118: 606-614, 2007

[7] Farman A, Somi MH, Sarami F, Farhang S, Five personality dimensions in patients with irritable bowel syndrome. Neuropsychiatr Dis Treat: 4, 959-962, 2008.

[8] Hoekstra HA, Ormel J, de Fruyt F (1996) NEO-PI-R - NEO FFI. Big five persoonlijkheidsvragenlijsten. Handleiding. Lisse: Swets \& Zeitlinger.

[9] Huskisson EC, Measurement of Pain. Lancet 9: 1127-1131, 1974.

[10] Jasper HH, The ten-twenty electrode system of the International Federation. J Electroenceph Clin Neurophysiol 20: 371-375, 1958.

[11] Kanda M, Matsuhashi M, Sawamoto N, Oga T, Mima T, Nagamine T, Shibasaki H, Cortical potentials related to assessment of pain intensity with visual analogue scale (VAS). Clin Neurophysiol 113: 1013-1024, 2002.

[12] Kemp AH, Hopkinson PJ, Stephan BC, Clark R, Gordon E, Bryant RA, Williams LM, Predicting severity of nonclinical depression: preliminary findings using an integrated approach. J Integrated Neuroscience 5: 89-110, 2006.

[13] Kovacs FM, Bagó J, Royuela A, Seco J, Giménez S et al, Psychometric characteristics of the Spanish versión of instruments to measure neck pain disability. BMC muscoloskelet Disord 9: 9-42, 2008.

[14] Labbé EE, Murphy L, O’Brien C, Psychological factors and prediction of headaches in college adults. Headache 37: 1-5, 1997.

[15] Lousberg R, Schmidt AJ, Groenman NH, The relationship between spouse solicitousness and pain behavior: searching for more experimental evidence. Pain 51: 75-79, 1992.

[16] Myin-Germeys I, Oorschot M, Collip D, Lataster J, Delespaul P, van Os J, Experience sampling research in psychopathology: opening the black-box of daily life. Psycholog Med 12: 1-15, 2009.

[17] Perneger TV, What's wrong with the Bonferroni Adjustments. British Medical Journal 316: 1236-1238, 1998.

[18] Schwartz L, Kraft GH, The role of spouse responses to disability and family environment in multiple sclerosis. American Journal of Physical Medicine \& Rehabilitation, 78: 525-532, 1999.

[19] Stowell H, Cerebral slow waves related to the perception of pain in man. Brain Research Bulletin 2: 23-30, 1977.

[20] Wall PD, Presynaptic control of impulses at the first central synapse in the cutaneous pathway. J Psychophysiol 188: 403-423, 1964.

[21] Zaslansky R, Sprecher E, Tenke CE, Hemli JA, Yarnitsky G, The P300 in evoked potentials. Pain 66: 39-49, 1996. 